\title{
Arquitectas. Estrategias y obstáculos de inserción de las primeras mujeres al campo de la Arquitectura argentina (primera mitad del siglo XX) ${ }^{1}$
}

\author{
Architect women. Strategies and obstacles of \\ insertion of the fist women into the Argentine \\ Architecture field (first half of the XXth century)
}

\author{
Natalia Silvina Daldi²
}

Fecha de recepción: 23-03-2018 - Fecha de aceptación: 23-07-2018

Hábitat y Sociedad (ISSN 2173-125X), n. . 11, noviembre de 2018, pp. 15-29.

http://dx.doi.org/10.12795/HabitatySociedad.2018.i11.02

\begin{abstract}
The History of Universal Architecture hardly ever has mentioned female architects's names who have worked in the disciplinary and professional field. Recent studies have dealt with the issue about women in relation to Architecture (work). However, none of them have specifically focused on the work insertion problematic of the first architect women into the Argentine architecture field during 1930's. Therefore, this paper aims to, on the one hand, explore two strategies which these first women developed to insert into the highly homogeneous male field and, on the other hand, identify the obstacles were encountered by them over the work insertion process. Thus, we analysed four graduated architect women in the 30's in the School of Architecture of University of Buenos Aires (UBA) as of conceptual categories by Pierre Bourdieu (1997) in "Theory of the action" (fields, habitus, capital, strategies) and, additionally, we have used other concepts about "Feminist theories" (symbolic violence, sexual division of labour).
\end{abstract}

\section{Key words}

Female architects; Architecture; History of Architecture; Professional field; Female work insertion; Feminism

\begin{abstract}
Resumen
La historia de la arquitectura universal, pocas veces, ha mencionado nombres de mujeres arquitectas que se hayan desempeñado en el campo profesional y disciplinar. Esta situación también se refleja en la Historiografía de la Arquitectura argentina. Si bien estudios recientes han abordado la temática de las mujeres en relación con la Arquitectura, ninguno se centra específicamente en la problemática de inserción de las primeras mujeres al campo. El presente trabajo pertenece a una investigación más amplia que busca comprender cómo fue el proceso de inserción de las primeras mujeres arquitectas al campo de la Arquitectura argentina durante la década de 1930. A partir de ello, este texto se propone, por un lado, explorar dos estrategias que desarrollaron ellas para ingresar a un campo altamente masculinizado y, por otro, identificar los obstáculos enfrentados durante el proceso de inserción. Para abordarlo, hemos analizado las experiencias de cuatro mujeres egresadas durante la década de los '30 de la Escuela de Arquitectura de la Universidad de Buenos Aires (UBA), a partir de las categorías teóricas desarrolladas por Bourdieu (1997) en Teoría de la Acción (campo, habitus, capital, estrategia), vinculadas con otras, propias de las Teorías Feministas (violencia simbólica, división sexual del trabajo).
\end{abstract}

\section{Pallabras clave}

Mujeres arquitectas; Arquitectura; Historia de la Arquitectura; Campo profesional; Inserción laboral de mujeres; Feminismo

\footnotetext{
1 Los primeros avances de esta investigación fueron presentados en las XIII Jornadas Nacionales de Historia de las Mujeres. VIII Congreso Iberoamericano de Estudios de Género "Horizontes revolucionarios. Voces y cuerpos en conflicto", realizado en Buenos Aires del 24 al 27 de Julio de 2017.

2 Arquitecta y doctoranda en Historia en la Facultad de Filosofía y Letras de la Universidad Nacional de Cuyo (Mendoza, Argentina); pertenece al equipo de investigación "Historia y Conservación patrimonial” del INCIHUSA, CONICET Mendoza, Argentina. Email: ndaldi@mendoza-conicet.gob.ar.
} 


\section{Introducción}

En Argentina, las arquitectas actuales no cuentan con referencias históricas que las identifiquen con su pasado femenino. Si bien la Arquitectura, desde los inicios, ha sido una profesión altamente masculinizada tanto en Europa como en América Latina, debido - entre varias cuestiones- a la falta de políticas de conciliación entre profesión y familia (Montaner y Muxí, 2015), a comienzos de siglo xx, algunas mujeres se animaron a romper el aislamiento de las aulas universitarias y comenzaron a estudiar la carrera. Posteriormente, ingresaron al campo disciplinar y profesional y ocuparon distintas posiciones dentro de él. Sin embargo, la Historia de la Arquitectura universal no ha dado cuenta de ello.

Muxí y Montaner (2015) sostienen que esto tiene que ver con una forma de construcción de la Historia que tiende a resaltar las figuras masculinas en detrimento de los logros y realizaciones femeninas. En consecuencia, las mujeres han quedado excluidas de la Historia de la Arquitectura canónica que, de alguna manera, las consideró poco relevantes en relación con los proyectos 'heroicos y extraordinarios' que realizaron los arquitectos varones, ubicándolas en un reducto pacífico de trabajo en la economía doméstica (ob. cit.). Este mecanismo invisibilizador también se refleja, aún hoy, en la historiografía de la Arquitectura argentina.

Si bien en Argentina, durante los últimos años, han comenzado a desarrollarse algunos estudios en torno a la visibilización y puesta en valor de las experiencias de mujeres arquitectas (Di Bello, 1997a; Moisset, 2013, 2017; Cirvini, 2015; Daldi, 2015, 2017, 2018, en prensa; Seri y Farré, 2018), aún no existen investigaciones científicas completas que se centren específicamente en el estudio del proceso de inserción de las primeras mujeres al campo, es decir, que hagan foco en 'ese proceso' que involucró múltiples aspectos. A partir de ello, el presente artículo se propone, por un lado, explorar dos estrategias que desarrollaron las primeras mujeres para ingresar al campo académico y profesional de la Arquitectura argentina: la elección de la carrera y la adquisición del habitus de los arquitectos. Por el otro, identificar algunos obstáculos enfrentados por ellas durante el proceso de inserción: violencia simbólica, división sexual del trabajo y discriminación por género.

Según Cirvini (2004), a principios de la década de 1930, el campo de la Arquitectura argentina estaba consolidado y era reconocido socialmente gracias a la participación activa de sus miembros, en su mayoría, varones. En efecto, desde la creación de la Escuela de Arquitectura $^{3}$ en 1901 hasta 1928, se habían recibido un total 485 arquitectos hombres y ninguna mujer. Recién en noviembre de 1929, egresó la primera: Finlandia Pizzul. Al cabo de dos años, egresaron de la Escuela dos mujeres más: Nelly Nieburh y María Luisa García Vouilloz. A ellas se siguieron sumando otras que elevaron el número de egresadas a 19 al finalizar la década de 1930, sobre 435 varones, sólo en ese periodo. Así, durante la década de los 30, la matricula femenina alcanzó un total de $4,36 \%$ frente a un $95,63 \%$ de matrícula masculina. ${ }^{4}$ Cabe destacar que a principios de la década de los años 30, la Escuela de Arquitectura de la UBA fue la única institución, dedicada a la enseñanza de la Arquitectura del país, de la que egresaron mujeres; y, al finalizar la década, fue la que mayor cantidad de mujeres egresó, manteniendo como institución cierta hegemonía en el egreso femenino. ${ }^{5}$ En este sentido, entendemos que las mujeres egresadas de esta institución durante la 
década de los treinta se constituyen en las "primeras arquitectas argentinas".

Para esta investigación, hemos seleccionado las experiencias de cuatro mujeres que estudiaron la carrera de Arquitectura en la Escuela de Arquitectura de la UBA durante la década de 1930 y que, luego de recibidas, ingresaron al campo disciplinar y profesional, ejerciendo su profesión por más de treinta años. Las experiencias seleccionadas corresponden a: la primera arquitecta argentina, egresada en 1929: Finlandia Pizzul; ${ }^{6}$ la cuarta arquitecta egresada en 1934, María Mercedes Arauz Obligado; ${ }^{7}$ la quinta arquitecta egresada en 1936, Stella Genovese ${ }^{8}$ y la egresada número diecinueve en 1939, María Alicia Anzorena. ${ }^{9}$ Cabe destacar que hemos seleccionado experiencias vividas en distintos periodos dentro de la década de 1930, para tratar el rango temporal en su totalidad y, así, poder encontrar similitudes y establecer diferencias entre ellas.

Partimos del supuesto que las primeras arquitectas, conscientes de la situación de desigualdad en la que se encontraban, producto de la visión androcéntrica de la época, desarrollaron un sistema de estrategias que les permitió ocupar distintas posiciones dentro del campo. Siguiendo a Bourdieu (1997), entendemos que estas mujeres, internalizaron los habitus de los arquitectos en función de poder transformarlo en un capital simbólico susceptible de inversión dentro del campo; y, a través de la illusio, entendida como condición y fundamento del juego, ejercieron sus prácticas.

Para cumplir con el objetivo de este artículo, hemos sistematizado las fuentes de nuestro corpus empírico (Revista de Arquitectura; ${ }^{10}$ Boletines oficiales y Carpetas de correspondencia entre los socios y la Sociedad Central de Arquitectos -SCA—; Notas en diarios de la época; Entrevistas a las primeras arquitectas realizadas por la Lda. Di Bello en 1997 ${ }^{11}$ y, a partir de ello, hemos establecido distintas variables de análisis en función de alcanzar el objetivo propuesto. Asimismo, hemos utilizado el Método Histórico (Topolsky, 1992) que sigue los siguientes pasos en el tratamiento de las fuentes: búsqueda, análisis, clasificación y sistematización de fuentes cuantitativas y cualitativas.

Si bien no existe una metodología específica para llevar a cabo una investigación de carácter feminista, nosotras abordamos varios conceptos y categorías propios de los llamados "estudios de género o estudios feministas" porque nos permiten develar cuestiones naturalizadas tradicionalmente en el orden social. Así, hemos adoptado "el punto de vista feminista" como punto de partida del proceso de investigación, que supone que "existen conceptos y categorías específicas que tendrán que entrar en acción si se lleva a cabo una investigación de carácter feminista" (Bartra, 2002, p. 148). Bajo esta premisa, abordamos la categoría "mujeres" a la manera beauvoireana de lo Otro. Beauvoir (1949) sostiene que la situación objetiva a las mujeres como lo Otro, reduciendo su libertad y su trascendencia a la inmanencia y a lo inescencial (cfr. Femenías, 2012, p. 19). No obstante, el concepto de género de Scott (1996) nos ayuda a comprender que el género es una construcción social y cultural, despojada de cualquier carga biológica.

Para referirnos a los hechos vividos por las primeras arquitectas durante el proceso de inserción al campo utilizamos la noción de 'experiencia' (Scott, 1992), la cual señala la condición política de un discurso declarado como neutral y permite darnos de cuenta de aquello que resulta excedente, subsidiario o invisible a la ciencia legitimada como tal. En este sentido, según Trebisacce (2016), la experiencia nos permi-
6 Finlandia Elisa Pizzul nació en Buenos Aires el 22 de mayo de 1902. Estudió la carrera de Arquitectura en la Escuela de Arquitectura de la UBA. Egresó en noviembre de 1929, convirtiéndose en la primera arquitecta argentina. Tuvo una destacada actuación profesional en el Ministerio de Salud Pública de la Nación. Además, fue miembro de la SCA. Falleció en Buenos Aires, en noviembre de 1987. 7 María Mercedes Arauz Obligado nació en Buenos Aires en 1908. Egresó de la Escuela de Arquitectura en 1934, siendo la cuarta arquitecta argentina. Trabajó en la Dirección de Arquitectura de la Municipalidad de La Plata (Buenos Aires), desde donde realizó múltiples proyectos de arquitectura estatal. Falleció en Buenos Aires a finales de la década de 1990. 8 Stella Elba Genovese nació en Buenos Aires en 1909. Egresó de la Escuela de Arquitectura en 1936, siendo la séptima arquitecta argentina. Trabajó como arquitecta de manera independiente y en asociación con otros arquitectos. Además, fue una destacada pintora.

9 María Alicia Anzorena nació en Mendoza aprox. en 1915. Egresó de la Escuela de Arquitectura en 1939, ocupando el numero dieciocho en la Nómina de egresados. Desarrolló la Arquitectura de manera independiente y en relación de dependencia con otros arquitectos. Ejerció la docencia universitaria en distintas cátedras de la Facultad de Arquitectura de la UBA. Falleció en Buenos Aires el 2 de enero de 2018.

10 La Revista de Arquitectura fue la publicación oficial de los miembros del Centro de Estudiantes de la Escuela de Arquitectura junto con la SCA. Tuvo una aparición mensual e ininterrumpida desde 1915 hasta 1951. Según Cirvini (2004), "en estos espacios se instaba al debate, se promovían conductas y modelaban las prácticas". En este sentido, la revista se constituye en una importante fuente documental ya que, a través de sus publicaciones, da cuenta de las transformaciones por las que atravesó el campo disciplinar a lo largo de ese periodo.

11 Estas entrevistas fueron realizadas en 1995 por la Lic. Roxana Di Bello (Argentina), quien entrevistó a dos arquitectas egresadas de la Escuela de Arquitectura (UBA) durante la década de 1930 con la metodología de la historia oral. Cabe destacar que, excepto estas entrevistas, no existen otra producción académica al respecto, y al no contar en la actualidad con las "voces vivas" de las protagonistas, las mismas se constituyen en fuentes primarias para nuestra investigación. Agradecemos especialmente a la Lic. Di Bello por haber aportado tan importante material para nuestra investigación. 
te dar testimonio de formas de dominio y opresión productora de sujetos subalternos (cfr. ob. cit., p. 289). Por último, hemos tomado otras nociones epistémicas como violencia simbólica y división sexual de las tareas que nos permiten complementar el enfoque. La violencia simbólica, por su parte, es una fuerza particular de la que disponen ciertos agentes sobre otros, mediante complicidad (cfr. Bourdieu, 2015, pp. 49-58). La división sexual de las tareas es una distribución arbitraria de las actividades asignadas a los géneros. Para Bourdieu (2015), tal división es una "construcción social arbitraria de lo biológico, del cuerpo masculino y femenino, de sus costumbres, de sus funciones y, en particular, de la reproducción biológica, en la cual se fundamenta, naturalizándola” (p. 22).

Por otro lado, el marco conceptual que utiliza Bourdieu en su Teoría de la acción (1997) nos permite comprender la relativa autonomía que tuvo el campo de la Arquitectura argentina en la primera mitad del siglo $\mathrm{xx}$, dentro del cual se insertaron las primeras mujeres. Para el autor, los campos sociales son como 'espacios de juego' históricamente constituidos con sus instituciones específicas y sus leyes de funcionamiento propias. Dentro del mismo, los jugadores o agentes interesados en el juego que allí se despliega (illusio) aceptan sus reglas y condiciones, es decir, comprenden el sentido del juego. Esto es lo que les permite a los jugadores (agentes) hacer infinidad de "jugadas", adaptadas a la infinidad de situaciones posibles, que ninguna regla podría prever. De este modo, el agente adquiere autonomía en el espacio de juego, y, a través del habitus como "disposición estratégica" de juego, realiza sus distintas jugadas.

Este artículo se ordena en cuatro partes. En primer lugar, desarrollamos una breve introducción con algunas nociones teóricas de nuestro marco conceptual para comprender aspectos generales relacionados con la constitución del campo de la Arquitectura en Argentina. En segundo lugar, describimos el escenario de la investigación haciendo hincapié en la situación de las mujeres argentinas hacia 1930; al mismo tiempo, exploramos dos estrategias de inserción que desarrollaron las primeras mujeres para ingresar al campo: la elección de la carrera y la adquisición del habitus de los arquitectos. En tercer lugar, identificamos algunos obstáculos que enfrentaron las mujeres al momento de la inserción desde el punto de vista feminista. Finalmente, adelantamos algunas conclusiones preliminares.

\section{Las primeras arquitectas}

\section{Argentina hacia 1930: Revolución femenina y pensamiento moderno}

12 Según Cirvini (2004), el proceso de modernización argentino se inició a mediados del siglo xix, con la organización constitucional del país, pero tuvo su más acelerado desarrollo entre 1880 y 1910. En algunos campos, como el de la arquitectura, sus efectos se prolongaron hasta promediar el siglo xx. Este programa político, socioeconómico y cultural, esencialmente transformador y progresista, se autoerigió como emancipador frente al pasado hispano-criollo asociado al atraso y a la ignorancia (cfr. p. 31).
En Argentina, a comienzos del siglo xx, la creciente urbanización y el impacto demográfico de la inmigración promovieron la aparición de nuevas tipologías de edificios. Así pues, la arquitectura pública adquirió una inusitada envergadura y la arquitectura privada se convirtió en un referente del status social y tuvo gran peso en la legitimación de un linaje entre los nuevos ricos donde abundaban los extranjeros. Consecuentemente, modernidad y modernización ${ }^{12}$ fueron imponiendo nuevas reglas al campo laboral. En este sentido, se ampliaron las demandas obreras y las mujeres se fueron sumaron con voz propia al incipiente mundo proletariado de las industrias. Así, se fueron convir- 
tiendo en piezas claves para el mejoramiento de la sociedad (Calvera, 1990).

Según Espegel (2004), hasta principios del siglo xx a las mujeres se les vetaba el ingreso a la universidad (cfr. p. 79). No obstante, las luchas sociales de carácter nacional e internacional y, entre ellas, las lideradas por los colectivos feministas, consiguieron que las mujeres accedieran a la enseñanza oficial. En 1910 y con motivo del centenario de la Revolución de Mayo, las primeras expresiones organizadas de feminismo argentino emprendieron dos importantes Congresos: el Congreso Patriótico de Mujeres y el Congreso Internacional Femenino. Estos eventos dieron como resultado la aprobación de propuestas más igualitarias en materia de derechos sociales, políticos y educacionales para las mujeres.

Así, hacia 1920, muchas mujeres se animaron a romper el aislamiento de las aulas universitarias y comenzaron a egresar de las universidades. Para esos años, las carreras de Medicina, Derecho, Filosofía e inclusive Ingeniería estaban egresando sus primeras mujeres (Lorenzo, 2016). La carrera de Arquitectura, a finales de la década de los '20 comenzó a obtener las primeras.

\section{Estrategias y recursos de inserción}

\section{La elección de la carrera}

En noviembre de 1905, la revista Arquitectura, ${ }^{13}$ publicó una nota titulada: "Una Mujer Arquitecto". En la misma, el autor de la nota, se preguntaba por qué en Argentina aún no había "mujeres arquitectos" si, hacia esos años, Buenos Aires ya contaba con doctoras en Medicina, en Filosofía y en Cirugía dental, o con escultoras y escritoras de fama consagrada como Lola Mora. Manifestaba que en Buenos Aires - como en todas las sociedades modernas de adelantada civilizaciónse veía ensanchar cada día el horizonte del campo de acción de la mujer; pero la ciudad no contaba aún con una mujer arquitecto, lo cual parecía ser la última expresión del feminismo. Decía: “‘acaso hay más inconvenientes para una mujer en dedicarse a la arquitectura que a la medicina por ejemplo?” (Chanourdie, 1905, p. 114).

Si bien en nuestro país el cambio de siglo ofreció, entre otras cuestiones, una visión un poco más renovada de algunas concepciones patriarcales que habían situado a las mujeres en posiciones pasivas dentro del orden social, ${ }^{14}$ lo cierto es que, hacia esos años, la Arquitectura aún no contaba con integrantes mujeres. En efecto, las pocas mujeres que para entonces se dedicaron a la arquitectura eran extranjeras, y ninguna de ellas se había recibido en nuestro país. ${ }^{15}$

Este fenómeno podría deberse a una simultaneidad de factores (políticos, sociales y culturales) que condicionaron la inserción de las mujeres al campo profesional y disciplinar de la Arquitectura. Entre estos factores, cabe mencionar que a mediados de la década de los años 20, momento en el cual comenzaron a ingresar las primeras mujeres a la Escuela de Arquitectura de la UBA, el campo de los arquitectos se encontraba en su etapa de consolidación, ${ }^{16}$ lo cual implicó un fortalecimiento institucional del gremio, así como una legitimación de la disciplina ante los poderes públicos y la sociedad en general (cfr. Cirvini, 2004, pp. 338-339).

No obstante, al momento de la publicación de la nota de Chanourdie (1905), la carrera de Arquitectura era de reciente inauguración
13 La revista Arquitectura era un suplemento especial y mensual que se editaba dentro de la Revista Técnica, la cual fue dirigida por Enrique Chanourdie entre 1904 y 1916.

14 Para Lobato (2007) una idea común en los análisis del trabajo ha sido el carácter pasivo de las mujeres. Sin embargo, Lobato considera que la idea de 'pasividad' no tiene en cuenta las peculiaridades con las que se desenvolvieron las experiencias laborales femeninas (cfr. pp. 191-192).

15 Según Gutiérrez (1994), hacia esos años, había algunas arquitectas extranjeras trabajando en Argentina. La arquitecta Nina Livia Viterbo fue una de ellas. Viterbo se recibió en la Escuela Superior de Arquitectura de Roma en 1926. Desde 1930, trabajó en Milán, Brasil y Argentina. Otra arquitecta extranjera, fue Sonia Golejewsky de Van Pevorgh quien egresó de la Escuela Superior de Arquitectura de París en 1934 y obtuvo su reconocimiento oficial para actuar profesionalmente en la Argentina en 1949.

16 Cirvini (2004) define cuatro etapas en el proceso de constitución del campo de los arquitectos argentinos. Estas etapas son: 1-Etapa crítica (1886-1901); 2-Etapa fundacional (1901-1910); 3-Etapa de consolidación (1910-1930) y 4-Etapa de expansión y crisis (1930-1948) (cfr. p. 338). 
(1901), el campo de los arquitectos se encontraba en su etapa de fundación y los agentes del campo buscaban diferenciar sus prácticas de la de los ingenieros (cfr. Cirvini, 2004, p. 90). En efecto, previo a la inauguración de la Escuela, la carrera funcionaba como una especialización de rango inferior dentro de la carrera de ingeniería (no tenía currícula propia). Por ello, la carrera de Arquitectura se fundó con el objetivo implícito de alcanzar una progresiva diferenciación respecto de la carrera de Ingeniería (ob. cit.).

La Escuela de Arquitectura fue fundada en 1901 en el seno de la Facultad de Ciencias Físicas, Exactas y Naturales de la Universidad de Buenos Aires (UBA), como un órgano dependiente de ella, lo cual aún las relacionaba directamente. Según Cirvini (2004), en nuestro país, el proceso de constitución del campo de la Arquitectura tuvo características particulares, a diferencia de algunos países de Europa. En Europa, las Escuelas de Arquitectura se fundaron en el siglo xix al amparo de las Academias, la formación era netamente artística y se proponían alcanzar una formación más técnica y científica adecuada a la época; mientras que en Argentina la Arquitectura nació como una rama de la Ingeniería a la que era necesario hacer crecer hasta adquirir independencia (cfr. ob. cit., p. 90).

Pese a los esfuerzos de diferenciación al interior del campo, para el imaginario colectivo de la sociedad, la carrera de Arquitectura aún era considerada una rama de la Ingeniería. Y la Ingeniería, hacia esos años, era considerada como una de las típicas "carreras masculinas", a las que las mujeres no podían acceder, probablemente debido a la cantidad de materias técnicas y científicas que la misma contenía. ${ }^{17}$

Esta podría ser una de las razones por las que, al momento de publicación de la nota, casi no había mujeres arquitectas ni estudiantes de Arquitectura en Argentina. Porque entonces el campo de los arquitectos se encontraba atravesando un proceso de diferenciación y especificación de la disciplina, respecto a la Ingeniería. Pero, al no estar aún superado el debate Ingenieros/Arquitectos, ${ }^{18}$ la Arquitectura también era considerada una carrera de hombres. En este sentido, se puede entender que la profesión no fuera de especial atención para las mujeres ya que, al ser asociada directamente con la ingeniería, también sería de exclusividad masculina.

Ahora bien, tras la inauguración de la Escuela, el campo disciplinar

17 En 1901, Elvira López decía en su tesis doctoral de filosofía, titulada El movimiento feminista: "Carrera muy poco tentada aún por las mujeres, y que realmente no es de las más apropiadas para su sexo, es la de Ingeniería”.

18 Para Cirvini (2004), el debate Ingenieros/Arquitectos fue uno de los más importantes debates fundacionales por los que atravesó el campo disciplinar y profesional de los arquitectos puesto que ambos colectivos buscaban delimitar el campo y especificarlo (cfr. pp. 80-81). 19 Para Stuar Hall, citado en Lobato (2007), la 'representación' es un proceso por el cual los miembros de una cultura usan el lenguaje para producir significados. Así pues, la literatura, la pintura, el cine y la fotografía son artefactos culturales que trabajan con materiales y elementos de la sociedad, crean actitudes, valores, conciencias e identidades (cfr. p. 284). de los arquitectos comenzó un importante proceso de diferenciación tanto de los habitus como de las prácticas respecto de la de los ingenieros; y, en este sentido, la formación artística que los arquitectos tenían funcionó como bandera de diferenciación. Así pues, los agentes del campo de la Arquitectura (tanto estudiantes como arquitectos recibidos) buscaron acentuar los rasgos distintivos de un habitus propio para diferenciarse de sus pares de la Ingeniería. En este sentido, se propusieron alimentar la representación imaginaria ${ }^{19}$ del arquitecto "artista", más libre y menos sujetos a convenciones de todo tipo, frente a la mentalidad práctica y científica de los ingenieros (cfr. Cirvini, 2004, p. 90).

Así fue que, a mediados de la década de los años 20, cuando el debate Ingenieros/Arquitectos había comenzado a perder fuerza producto de haber cumplido ya una función diferenciadora y aglutinadora en un "nosotros" para los arquitectos (cfr. ob. cit., p. 95), las primeras estudiantes comenzaron a ingresar a la Escuela. Probablemente, esta coincidencia tenga que ver con que la carrera de Arquitectura, al haberse afianzado con un perfil más artístico que técnico, se presentaba frente a la sociedad como una opción más apropiada para las mujeres. De 
esto dan cuenta los testimonios de Finlandia Pizzul y Mercedes Arauz Obligado.

En una entrevista realizada luego de recibida —en 1929- por el diario El Argentino, Pizzul manifestó que había decidido estudiar Arquitectura porque le tomó afición al dibujo cuando estaba en el colegio secundario y entendía que la carrera tenía especial relación con el dibujo:

(...) durante mis estudios en el liceo le tomé tal afición al dibujo - tenía una excelente profesora en esa materia- que, al correr el tiempo, sobresalía en esa especialidad. Y del dibujo a la arquitectura no hubo más que un paso (Pizzul, citada en Pozzo Ardizzi, 1930, p. 10).

Por su parte, Arauz Obligado, ante la pregunta de por qué había decidido estudiar Arquitectura, le expresó a Di Bello (1997b) que si bien a ella le gustaba Ingeniería su padre la había inscripto en Arquitectura porque la consideraba más adecuada para una mujer:

A mí me gustaba Ingeniería, y el que me fue a inscribir fue papá, y cuando vuelvo me dice: "Te he inscripto en Arquitectura." - "¿Y por qué en Arquitectura?" - "Porque me parece mejor para una mujer" (Arauz Obligado, citada en Di Bello, 1995, p. 7).

Siguiendo a Palermo (2006), quien sostiene que la elección de la carrera es un momento clave dentro del proceso de profesionalización, podemos comprender que la elección de la carrera de Arquitectura por parte de estas mujeres tuvo que ver con que la misma se adaptaba mejor a las expectativas de lo que se consideraba "más adecuado" para una mujer en su época: pues, elegir una carrera con una orientación más artística que técnica sería la clave para que una mujer de su época desarrolle una profesión y la ejerza con éxito, sin alterar completamente los roles y mandatos sociales preestablecidos para el estereotipo de género femenino de la década de $1920 .{ }^{20}$ En este sentido, tal elección se convierte en una estrategia de inserción al campo en tanto que la carrera podría ser ejercida con mayor facilidad al adaptarse mejor a los roles de género femenino del momento.

Para Gluzman (2016), en Argentina, el trabajo remunerado femenino fuera del hogar se convirtió en una de las preocupaciones centrales del periodo (cfr. pp. 50-51). Así pues, los debates sobre la educación femenina se imbricaban frecuentemente con la discusión de las áreas 'naturalmente' más aptas para el desarrollo laboral de las mujeres. En tal sentido, se concluía que las bellas artes eran el terreno más favorable para la actividad de las mujeres ya que les permitiría llevar adelante un hogar. De este modo, a finales del siglo XIX, muchas mujeres comenzaron a ver el arte como una pasión que también podría ser un trabajo rentable.

Por otro lado, siguiendo a Palermo (2006), vemos que en la elección de la carrera y en los proyectos profesionales de las mujeres se entrecruzan diversos factores que pueden afectar la posibilidad de ingresar a una institución de educación superior y dar continuidad a los estudios hasta graduarse (cfr. p. 46). Estos factores incluyen: las expectativas de los padres, el apoyo y estímulo de la familia, los compañeros y los docentes del colegio secundario, entre otros. En este sentido, las experiencias de Genovese y Anzorena testimonian la influencia fami-
20 Para Lobato (2007:105-108), a finales del siglo xix se estaba afirmando el ideal de madre de familia, reina y señora de la vida doméstica. Posteriormente, a comienzos del siglo $\mathrm{xx}$, las representaciones de las mujeres modernas enfatizaban en el hogar una auténtica transformación del trabajo doméstico y la mujer "moderna," que era sinónimo de actual, se la asociaba a la tecnología, es decir, al uso de artefactos domésticos. Asimismo, se la relacionaba con el cuidado de los otros. 
liar - especialmente masculina - en la orientación de sus elecciones profesionales.

Genovese, al momento de estudiar Arquitectura, tenía un hermano mayor que era arquitecto y había egresado años antes de la Escuela de Arquitectura de la UBA; a su vez, estaba casada con el arquitecto Carlos Baldini Garay —egresado de la misma institución en 1930—, quien era miembro destacado de la SCA e integrante del comité editorial de la Revista de Arquitectura. Anzorena, por su parte, manifestó a Di Bello (1997b) que ella eligió la carrera porque tenía un novio que también la estudiaba:

Tenía un novio que entonces estudiaba Arquitectura ( ) y, entonces, él era tan entusiasta de la Arquitectura, decía: "voy a hacer esto y voy a hacer aquello", yo le digo: - "Voy a seguir Arquitectura." ——De ninguna manera, las mujeres no pueden seguir Arquitectura." - “Sí, voy a seguir Arquitectura” y, para llevarle la contra, seguí Arquitectura (Anzorena, citada en Di Bello, 1996, p. 378).

Estas experiencias nos permiten comprender otros factores que ejercieron influencia en la elección de la carrera ya que, para Palermo (2006), las mujeres tienen buenas razones para escoger alternativas que se adapten mejor al lugar que se les ha asignado en la familia y en la sociedad y estas elecciones son producto de estrategias conscientes y razonadas.

\section{La adquisición del habitus académico}

Como ya mencionamos, la creación en 1901 de la Escuela de Arquitectura en el seno de la Facultad Ciencias Físicas, Exactas y Naturales de la UBA, señaló un paso decisivo y trascendental en la formación profesional y especializada de los arquitectos. Esto significó, por un lado, la posibilidad de autonomía en la formación de profesionales de grado respecto de la Ingeniería y, por otro, permitió gradualmente modelar las prácticas acordes con una realidad propia, distinta a la europea. En este sentido, el ámbito universitario fue el sitio privilegiado de la formación especializada e 'inculcación' del habitus profesional a los arquitectos (cfr. Cirvini, 2004, p. 313).

En efecto, la Escuela de Arquitectura, desde el momento de su inauguración, buscó diferenciar su Plan académico del de la carrera de Ingeniería a través de la incorporación de materias de formación artística y talleres (cfr. ob. cit., p. 289).$^{21}$ En 1915, el mencionado Plan sufrió otras modificaciones en función de perfeccionar la 'inculcación' del habitus a los alumnos y reforzar la formación artística que ellos tenían (cfr. ob. cit., p. 292). Así pues, la enseñanza académica se apoyó tanto en el dominio del dibujo como en un profundo conocimiento de los estilos artísticos (cfr. ob. cit., p. 290). Años más tarde, en 1929, se incrementó la carga horaria de materias y se incorporó el Urbanismo en tercer año. En 1934, se adicionó un año más de formación en la carrera - el $6^{\circ}$ año- (Di Bello, 1997b); no obstante, las cuatro experiencias analizadas quedaron incluidas dentro del Plan de 1929 que contemplaba una formación de sólo cinco años.

A principios de la década de los treinta, cuando comenzaron a ingresar las primeras mujeres a la Escuela de Arquitectura, el habitus académico de los arquitectos ya estaba 'naturalizado' y podía diferenciarse claramente del habitus de los ingenieros (cfr. Cirvini, 2004, p. 294). 
El reglamento de ingreso a la Escuela establecía que, para entrar, los estudiantes debían poseer título de bachiller y rendir un examen de ingreso. Sólo estaban eximidos de rendir el examen los egresados del Colegio Nacional de Buenos Aires (que eran todos varones) y el Instituto Libre de Segunda Enseñanza por poseer planes de estudios de seis años (cfr. Di Bello, 1997b, pp. 37-38). En este sentido, las mujeres quedaban obligadas a rendir el examen ya que, la mayoría de ellas, eran egresadas del Liceo de Señoritas. ${ }^{22}$ De esto dan cuenta las experiencias analizadas en esta investigación.

El examen de ingreso hasta 1930 se componía de las siguientes asignaturas: Matemáticas, Geometría del espacio, Dibujo de ornato e Historia de la civilización. Cada una de estas materias constituían a su vez instancias evaluativas independientes con carácter eliminatorio, una sobre la otra. ${ }^{23}$ Esto significaba que, si los estudiantes eran aplazados en cualquiera de las asignaturas, automáticamente debían volver a rendir todas las materias —incluso las aprobadas previamente-. Luego en 1934, el examen dejó de ser eliminatorio.

Mercedes Arauz Obligado, era egresada del Liceo de Señoritas y rindió su examen de ingreso aproximadamente en 1927. Relata su experiencia en la entrevista realizada por Di Bello en 1995:

\section{¿Cómo era ese examen de ingreso, se acuerda?}

Bueno, me acuerdo que había examen de Historia, a mí que me gustaba tanto la Historia. Lo pasé, no sé cómo di Historia y después había Matemáticas, ponían unos problemas de Matemáticas y uno los tenía que resolver, de Aritmética y Geometría.

\section{¿Y de dibujo tenían?}

Y después sí un examen con otro, de Dibujo Lineal, algunas cosas ( ) que nos puso un profesor.

Pero con la preparación que Ud. tenía del Liceo

Con eso bastaba. Sobre todo, que a mí me había gustado mucho, con Nelly Seeber preparábamos unas carpetas de dibujo, de clases de dibujo, según cómo se nos ocurriera. Y mamá me ayudaba mucho, porque a ella le gustaba también, así que muchos estaban hechos por mí, la mitad hechos por ella (Arauz, citada en Di Bello, 1995, p. 10).

Alicia Anzorena, también fue egresada del Liceo de Señoritas y rindió su examen de ingreso en 1933, describiéndolo de esta manera:

$\dot{¿}^{Y}$ usted tuvo que rendir ingreso para entrar?

Claro. Ahí dice... (Se refiere al Certificado de Estudios)

¿Y cómo era ese ingreso?

Era bastante jorobado. Teníamos que dar Historia de la Civilización, ahí saqué cuatro, no saqué ocho, miento. Dibujo lineal, cuatro, Geometría, ocho, Aritmética y Álgebra, nueve, y Física, diez. Fíjate vos, quiere decir que no debería haber seguido Dibujo, Dibujo, cuatro (risas)

A lo mejor era el examen más exigente

Era Dibujo lineal, claro, yo no tenía la menor idea, porque yo hacía dibujo a mano levantada y resulta que ahí nos ponían ( ) cómo se llamaba, un tiralíneas y teníamos que hacer líneas paralelas, líneas a $45^{\circ}$, cruzadas a otros $45^{\circ}$, en tinta china, ( ) teníamos que regular el tiralíneas, era un espanto

¿Y usted se preparó para rendir ese examen?

Sí, claro que me preparé. Me preparé en las materias esas teóricas, pero en el dibujo, no tenía la menor idea ( ) (Anzorena, citada en Di Bello, 1996, p. 381).
22 El Liceo Nacional de Señoritas $\mathrm{N}^{\circ}$ de Capital Federal (Buenos Aires) fue una institución prestigiosa que brindaba educación secundaria sólo a mujeres. 23 Posteriormente, en 1934, se unificaron todos los exámenes de ingreso a la Facultad de Ciencias Físicas, Exactas y Naturales de la UBA. El nuevo examen general costaba de dos pruebas orales, a excepción de la arquitectura, para la cual, la primera prueba sería práctica sobre dibujo lineal y natural. Y la segunda, sería aritmética, geometría, algebra y física. Además, el carácter eliminatorio del examen de ingreso se modificó (por ser considerado anacrónico) y se suprimió el examen de Historia de la civilización (Di Bello, 1997b:42). 
Figura 1: "Una entrada a cochera", trabajo realizado por la alumna Stella Genovese en 1931 para la materia Arquitectura de $2^{\circ}$ curso que dictaba el profesor René Karman. Fuente: Revista de Arquitectura $\mathrm{n}^{\circ} 127,1931$, p. 357.
24 Para un abordaje más específico sobre el tema, véase: Daldi, N. (2015), Las primeas arquitectas. Logro académicos y género en la Revista de Arquitectura (1926-1947). Ponencia en I Congreso Nacional e Internacional de Historia del Arte, Cultura y Sociedad. Discurso, poder e ideologías en las artes en Latinoamérica, UNCuyo (Mendoza, Argentina); Daldi, N. (en prensa), La participación de las primeras arquitectas en la Revista de Arquitectura (Argentina, 1926-1947). Área [Manuscrito enviado el 11 de junio de 2018 para el Dossier 24].

25 Según Valentino (2004) citado en Cravino (2015), el modelo Beaux Arts establecía que: "los concursos mensuales forman parte esencial en la formación del estudiante. Es obligatorio presentarse como mínimo a dos cada año. Los programas son variados y están definidos por los profesores de teoría. Generalmente están referidos a temas de arquitectura pública, religiosa o a arquitectura privada y su decoración. Plantean distintos niveles de complejidad, siendo los más megalómanos los correspondientes al concurso anual que otorga el Prix Rome, consagración máxima para cualquier profesional" (p. 124).

26 En 1934 se realizaron dos concursos anuales: uno fue en el mes de abril y el otro en el mes de diciembre. En el primer concurso, las mujeres que obtuvieron premios, y que luego salieron mencionadas en la revista, fueron: Ítala Fulvia Villa y Blanca Hirsh, que se destacaron en los segundos y terceros lugares de la materia Arquitectura de V año; Julia Molina y Vedia obtuvo el primer premio en la materia Composición Decorativa del $2^{\circ}$ curso. Posteriormente, en diciembre, Celina Dubin obtuvo el primer premio en la materia $A r$ quitectura de $2^{\circ}$ curso, mientras que Julia Molina y Vedia obtuvo el primer premio en la materia Arquitectura de 3er curso.

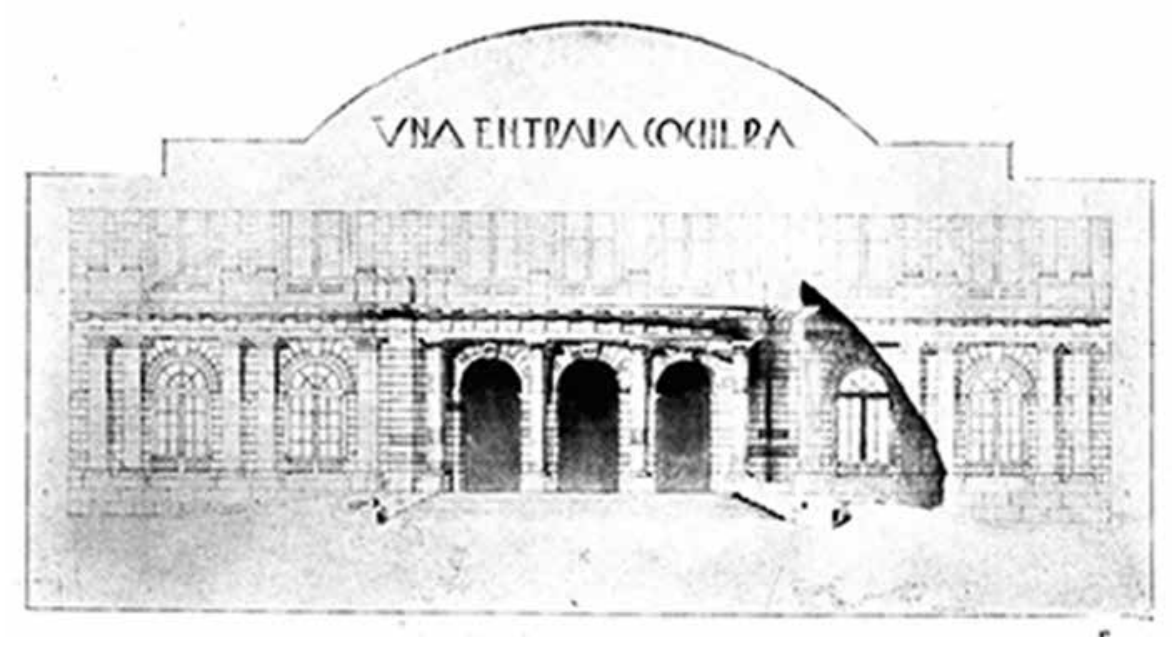

Sin lugar a dudas, estas instancias evaluativas fueron el primer acercamiento que tuvieron estas mujeres tanto a las prácticas disciplinares como al habitus de los arquitectos. En este sentido, entendemos que, si bien el examen de ingreso pretendía funcionar como filtro 'vocacional' de los aspirantes a la carrera, también buscó de alguna manera modelar el conjunto de habilidades y destrezas que traían (o no) los alumnos, en función de ser volcados luego a las prácticas académicas dentro de la Universidad. De esta forma, la adquisición desde el inicio del habitus académico de los arquitectos les permitiría a las primeras estudiantes desarrollar mejores jugadas dentro de su trayectoria educacional.

A través de las publicaciones que hacía la Revista de Arquitectura, se ha podido observar cómo fueron adquiriendo el habitus académico las primeras estudiantes dentro de la carrera (Daldi, en prensa). ${ }^{24}$ Pues, en la sección "Trabajos de la Escuela de Arquitectura" de la mencionada revista, se difundían con frecuencia los proyectos de los alumnos destacados y las mujeres aparecen mencionadas en reiteradas ocasiones. Ejemplo de ello es la Figura 1, la cual da cuenta, por un lado, del buen desempeño académico que ellas tenían ya que se publicaban sólo los proyectos de alumnos sobresalientes, y, por el otro, el grado de apropiación de estas alumnas, tanto de las teorías como de las prácticas académicas.

Anualmente los profesores de la Escuela organizaban los Concursos Anuales de Arquitectura y Composición Decorativa al mejor estilo Beaux Arts. ${ }^{25}$ Estos concursos, eran una práctica evaluativa que se realizaba anualmente bajo la modalidad de 'encierro'. Así pues, el famoso "encierro" consistía en la ejecución de un proyecto, que debía ser realizado por los alumnos en un transcurso de ocho horas, exclusivamente, dentro de la Universidad (Di Bello, 1997b). Los estudiantes se veían obligados a plasmar todas sus destrezas y conocimientos proyectuales (tanto teóricos como prácticos) en función de obtener algún reconocimiento meritorio en el concurso. La Revista de Arquitectura daba a conocer los nombres de los alumnos ganadores y publicaba las láminas de los proyectos ganadores.

A través de la mencionada revista hemos podido conocer los nombres de las mujeres que obtuvieron premios y menciones honorificas en los concursos anuales realizados durante la década de 1930. En efecto, hemos podido constatar que la Revista de Arquitectura mencionó por primera vez en 1934 a mujeres que obtuvieron premios en los concursos realizados ese mismo año. ${ }^{26}$ En 1935, la única estudiante mujer 
que recibió premios fue Julia Molina y Vedia. ${ }^{27}$ En 1936, se destacó en el concurso anual Alicia Anzorena que obtuvo una mención especial ${ }^{28}$ por un proyecto denominado "una Estela Funeraria”, realizado para la materia Composición Decorativa. El mismo, fue difundido ese mismo año por la Revista de Arquitectura.

En suma, a través de la 'completa' participación de las mujeres en las propuestas curriculares y académicas de la Escuela de Arquitectura hemos podido observar cómo el habitus académico —entendido como un sistema de disposiciones adquiridas-, fue incorporado por las estudiantes y se convirtió en una disposición estratégica en la medida en que les permitió formar parte del grupo estudiantil, hablar el mismo lenguaje, y realizar las mismas prácticas. Además, el habitus adquirido, aumentó el capital cultural de estas mujeres.

\section{Obstáculos enfrentados}

Si bien, como vimos antes, la carrera de Arquitectura históricamente había sido considerada una carrera de hombres, a finales de la década de los 20 está concepción comenzó a cambiar. Algunos testimonios de la década de los años treinta entrevistados por Di Bello en 1997, manifiestan que:

(...) La entrada de la mujer trajo una cosa, varias ventajas. Una de ellas era que las mujeres entraron con un nivel de educación y de corrección mejores que los chicos ( ) Y la enseñanza también, la entrada de las mujeres permitió acaparar, tomar más... más dimensiones, nuevas dimensiones y entrar en trabajos más interesantes (Di Bello, 1997ª , párr. 23).

Las experiencias analizadas nos muestran que el orden social patriarcal se manifestaba a través de varias cuestiones. Según Palermo (2006), las jóvenes que eligen carreras técnicas son buenas estudiantes y saben que la carrera elegida proporciona estatus y mayores oportunidades laborales (cfr. p. 32). Sin embargo, a lo largo de su trayectoria educativa se encuentran con innumerables dificultades u obstáculos, que no se les presentan a sus compañeros. Algunas de estas dificultades son: falta de modelos de identificación; sentimiento de ser consideradas por la sociedad menos femeninas; necesidad de controlar el futuro profesional, lo que hace que se culpen a sí mismas por las dificultades que anticipan que tendrán en su carrera profesional; percepción de que la valoración de familiares, profesores y estudiantes varones hacia su elección de carrera es baja; etc.

Como mencionamos antes, en las experiencias analizadas la elección de la carrera se ve influenciada por la opinión de algún miembro masculino de la familia (generalmente, el padre y, en otros casos, el novio o hermano), porque la consideraban "una carrera más apropiada para una mujer". ${ }^{29}$ Siguiendo a Bourdieu (2015) — quien sostiene que "el mundo del trabajo está repleto de pequeños compartimentos profesionales que funcionan como unas cuasi-familias en las que el jefe de servicio, casi siempre un hombre, ejerce una autoridad paternalista,
Figura 2: "Una Estela Funeraria", proyecto realizado por la estudiante María Alicia Anzorena para el Concurso anual de Arquitectura y Composición Decorativa de 1936. Fuente: Revista de Arquitectura $n^{\circ} 192$, diciembre de 1936, pp. $613-615$.
27 Julia Molina y Vedia obtuvo el tercer premio en la materia Composición decorativa del $2-^{\circ}$ curso, y una mención especial en la materia Arquitectura de $4^{\circ}$ curso. 28 La mención especial era equivalente a obtener un cuarto premio.

29 Véase el testimonio de Arauz Obligado en la estrategia de la elección de la carrera citado anteriormente. 
basada en la envoltura afectiva, ofreciendo una protección generalizada a un personal subalterno fundamentalmente femenino" (p. 77)—, entendemos que la influencia en la elección de la carrera por parte de la autoridad paternal sobre las estudiantes reproduce la división sexual de las tareas, asignándole a estas mujeres papeles socialmente distribuidos en la estructura socio-patriarcal.

Para el autor, el orden social tiende a ratificar la dominación masculina en la cual se apoya a través de la división sexual del trabajo (ob. cit.). Sin embargo, esta división opera a través de un mecanismo denominado violencia simbólica. Así pues, la violencia simbólica funciona, por un lado, a través de una 'creencia' implícita, involuntaria, y no elegida por el individuo, y por el otro, como un derecho de entrada a un juego producto de una pertenencia a un determinado espacio de juego (cfr. ob. cit., pp. 49-58). Consideramos que la violencia simbólica fue ejercida hacia las mujeres en tanto que la autoridad paternal —que elige por ellas la carrera según una concepción androcéntrica- les ofrece una cierta "protección" que parece "natural", a la cual ellas adhieren inconscientemente, reproduciéndose así nuevas relaciones sociales de dominación.

Por otro lado, pese al ambiente eminentemente moderno que habitaba en la Escuela de Arquitectura hacia esos años, algunas experiencias manifiestan la presencia de ciertas "reglas implícitas" relacionas con un pensamiento patriarcal y diferenciador que las condenaba por su condición femenina, discriminándolas por el género y limitándolas al ejercicio de ciertas acciones:

Cuando yo entré había 150 muchachos, y la única mujer fui yo, entonces se hizo una votación para nombrar al delegado del Consejo, me nombraron a mí delegada, ¿no? Y el Consejo dijo que mujeres no, tenía que renunciar y volver a llamar a otra votación. Dijeron: -'no, no, de ninguna manera la mujer, no (Anzorena, citada en Di Bello, 1996, p. 367).

Además, algunos compañeros veían la llegada de las mujeres como una 'competencia' lo cual reforzaba aun más la discriminación por el género:

Todos me adoraban porque yo era la única chica, pero cuando les empecé a ganar concursos, era un odio que me tomaron y empezaron las competencias. (...). Había mucha competencia, ya te digo que cuando les gané los concursos me reventaron (Anzorena, citada en Di Bello, 1996, p. 386).

Por su parte, algunos profesores ejercían violencia simbólica a través del hostigamiento verbal hacia las estudiantes, de esta forma se reflejaba el pensamiento patriarcal y machista que habitaba para entonces en la Escuela:

Había un profesor que no sé cómo se llamaba, que por suerte me olvidé el nombre, que era misógino, y entonces a las mujeres nos reventaba. Era un ingeniero, insoportable. Todas las mujeres sabes qué teníamos que transpirar pero sangre para pasar. Decía que las mujeres no teníamos que estar en la facultad. Teníamos que luchar contra eso ¿no? También tenía otro en métrica, el ayudante, que dijo: - 'ustedes deberían estar haciendo calceta y lavando platos'. Asique tenías que luchar contra esa bestialidad, los tipos eran insoportables (Anzorena, citada en Di Bello, 1996, p. 387). 
En relación a esto, sostenemos que la estructura jerárquica socialpatriarcal que distribuía sexualmente las tareas, dentro y fuera de la Escuela de Arquitectura, sentenciaba aquellas tareas que eran realizadas por fuera de la lógica androcéntrica, la cual consideraba que las posiciones dominantes siempre debían ser cuestión masculina, y el resto debía estar subordinado.

\section{Conclusiones preliminares}

Como la mayoría de las profesiones liberales, la Arquitectura había sido históricamente una carrera de hombres. En nuestro país, fue recién a mediados de la década de los veinte cuando las primeras mujeres se animaron a romper el aislamiento de las aulas universitarias de la Escuela de Arquitectura de la UBA. Sin embargo, cuando las mujeres comenzaron a incorporarse, advirtieron en seguida que la Institución aún no estaba preparada para recibirlas, tanto en términos funcionales como ideológicos.

Esto puede traducirse tanto en tener que dirigirse al edificio de Ciencias exactas para utilizar los sanitarios, porque el edificio donde se cursaba la carrera no tenía baños para mujeres, como el pensamiento androcéntrico que reproducía la estructura social-patriarcal de la época y las condenaba por su condición femenina. Sin embargo, como vimos, las primeras arquitectas desarrollaron diversas estrategias para superar los obstáculos que se presentaron tanto al momento del ingreso a la Universidad como a la hora de la inserción al campo disciplinar y profesional.

La estrategia de "elegir Arquitectura" y no otra carrera técnica como la Ingeniería, les permitió desarrollar su experiencia académica con mayor fluidez ya que, desde sus años en el Liceo las estudiantes se sentían motivadas y atraídas por materias de dibujo artístico, y consiguientemente, la carrera les permitiría desarrollar aún más esa veta. Por otro lado, la estrategia de "apropiación" de los habitus de los arquitectos las dotó de un sistema de herramientas simbólicas susceptibles de ser utilizadas dentro del campo. Además, la adquisición de los habitus aumentó el capital cultural y simbólico de estas mujeres y contribuyó a fortalecer la illusio de las nuevas agentes del campo. 


\section{Referencias}

Bartra, E. (2002). Reflexiones metodológicas. En Bartra, E (ed.), Debates en torno a una metodología feminista. México: UNAM, pp. 141-158.

Bourdieu, P. (1997). Razones prácticas Sobre la teoría de la acción. Traducción de Thomas Kauf. Barcelona: Anagrama. Edición original en francés, Raisons pratiques. Sur la théorie de l'action. París, Éditions du Seuil, 1994. Recuperado el 12 de agosto de 2018, de: http://epistemh.pbworks.com/f/9.\%2BBourdi eu\%2BRazones\%2BPr\%C3\%A1cticas.pdf.

Bourdieu, P. (2015). La dominación masculina. Barcelona: Anagrama. Edición original en francés, La Domination masculine. París, Éditions du Seuil, 1998.

Calvera, L. (1990). Mujeres y feminismo en Argentina. Buenos Aires: Grupo Editor Latinoamericano.

Chanourdie, E. (1905). Una Mujer Arquitecto. Revista Técnica, 32, 114-116.

Cirvini, S. (2004). Nosotros los Arquitectos. Campo disciplinar y profesión en la Argentina moderna. Mendoza: CRICIT / INCIHUSA.

Cirvini, S. (2015) Colette Boccara. La trayectoria singular de una mujer "arquitecto". Registros, 12, 41-52. Recuperado el 12 de agosto de 2018, de: https:/ / revistasfaud.mdp.edu.ar/registros/issue/view/11.

Cravino, A. (2015). Enseñanza de la Arquitectura. Una aproximación histórica. 1901-1955. La inercia del modelo Beaux Arts. Buenos Aires: Nobuko.

Daldi, N. (2015) Las primeas arquitectas. Logro académicos y género en la Revista de Arquitectura (19261947). En Discurso, poder e ideologías en las artes en Latinoamérica, I Congreso Nacional e Internacional de Historia del Arte, Cultura y Sociedad. Mendoza: UNCuyo.

Daldi, N. (2017). Beatriz Marta Carlota Penny Cánovas. En Raffa, C. (ed.) Arquitectos en Mendoza. Biografias, trayectorias profesionales y obras (1900-1960), volumen 1. Mendoza: EDIFYL- UNCuyo, pp. 185-186.

Daldi, N. (2018, mayo) Las modernas argentinas. Algunas producciones artísticas de las primeras arquitectas (primera mitad del siglo xx). En VIII Encuentro de docentes, investigadores en Historia del diseño, la arquitectura y la ciudad. Facultad de Arquitectura, Urbanismo y Diseño, sede Ciudad Universitaria, Universidad Nacional de Córdoba, Córdoba, Argentina.

Daldi, N. (en prensa). La participación de las primeras arquitectas en la Revista de Arquitectura (Argentina, 1926-1947) Área, [Manuscrito enviado el 11 de junio de 2018].

de Beauvouir, S. (1949). Le Deuxième Sexe. París: Gallimard.

Di Bello, R. (1995, 6 de diciembre). [Entrevista a la Arq. María Mercedes Arauz Obligado]. La Escuela de Arquitectura y los egresados de la década del '30: formación, ideología y praxis, pp. 1-22. Buenos Aires: Instituto de Arte Americano e Investigaciones Estéticas "Mario J. Buschiazzo" / Facultad de Arquitectura, Diseño y Urbanismo.

Di Bello, R. (1996, 18 de octubre). [Entrevista a la Arq. María Alicia Anzorena]. La Escuela de Arquitectura y los egresados de la década del '30: formación, ideología y praxis, pp. 1-22. Buenos Aires: Instituto de Arte Americano e Investigaciones Estéticas "Mario J. Buschiazzo" / Facultad de Arquitectura, Diseño y Urbanismo.

Di Bello, R. (1997a). La presencia femenina en las aulas de la UBA: las primeras arquitectas. En II Encuentro Nacional "La Universidad como Objeto de Investigación”. Centro de Estudios Avanzados (CEA), Universidad de Buenos Aires, Argentina. Recuperado el 12 de julio de 2018, de: http:/ /www.equiponaya.com.ar/congresos/contenido/cea_1/3/23. htm.

Di Bello, R. (1997b). La Escuela de Arquitectura y los egresados de la década del '30: formación, ideología y praxis (Segundo Informe anual de la Beca de Iniciación). Buenos Aires: Instituto de Arte Americano e Investigaciones Estéticas "Mario J. Buschiazzo" / Facultad de Arquitectura, Diseño y Urbanismo

Femenías, M. L. (2012). Sobre sujeto y género. (Re) Lecturas feministas desde Beauvoir a Butler (2. ${ }^{\mathrm{a}}$ ed.). Buenos Aires: Prohistoria.

Gluzman, G. G. (2016). Trazos invisibles. Las mujeres artistas en Buenos Aires (1890-1923). Buenos Aires: Biblos.

Gutiérrez, R. (1994), Las primeras arquitectas. En Sociedad Central de Arquitectos (ed.), Sociedad Central de Arquitectos, 100 años de compromiso con el país 1886-1986. Buenos Aires: Sociedad Central de Arquitectos, p. 176.

Gutiérrez, A. (2005). Las prácticas sociales: una introducción a Pierre Bourdieu. Córdoba: Ferreyra.

Harding, S. (1998). ¿Existe un método feminista? Traducción de Gloria Elena Bernal. Edición original en inglés, Is There a Feminist Method? En Harding, S. (ed.), Feminism and Methodology. Bloomington (Indianapolis): Indiana University Press, 1987. Recuperado el 9 de abril de 2018, de: http://investiga.uned.ac.cr/cicde/images/metodo.pdf.

Lobato, M.Z. (2007). Historia de las trabajadoras en la Argentina (1869-1960). Buenos Aires: Edhasa.

López, E. (2009). El movimiento feminista. Primeros trazos del feminismo en Argentina. Buenos Aires: Biblioteca Nacional.

Lorenzo, M.F. (2016). Que sepa coser, que sepa bordar, que 
sepa abrir la puerta para la universidad. Buenos Aires: Eudeba.

Moisset, I. (2013). Marina Waisman y la investigación. MW. Revista del Centro Marina Waisman de Formación de Investigación en Historia y Crítica de la Arquitectura, 12, 13-27.

Moisset, I. (2017). Cien Arquitectas en Wikipedia. Dearq, 20, 20-27. doi:10.18389/dearq20.2017.02.

Montaner, J. M. y Muxí, Z. (2015). La Construcción del Relato arquitectónico. Summa+, 143, 112-113. Recuperado el 12 de agosto de 2018, de: http:/ / www.revistasummamas.com.ar/revista_pdf/143/116\#visor.

Palermo, I. (2006). Mujeres y elecciones de carreras no tradicionales. El caso de la Universidad de Luján (Tesis doctoral). Facultad de Filosofía y Letras (UBA), Buenos Aires, Argentina. Recuperado el 12 de agosto de 2018, de: repositorio.filo.uba.ar/jspui/bitstream/filodigital/1867/1/uba_ffyl_t_2006_825792.pdf.

Pozzo Ardizzi, L. (1930). La argentina tiene ya su primera mujer arquitecto. El argentino, pp. 10 y 14.
Scott, J.W. (1992). Experiencia. En Butler, J. y Scott, J.W. (eds), Feminists Theorize the Politica. New York: Routledge, pp. 42-73.

Scott, J.W. (1996). El género: una categoría útil para el análisis histórico. En M. Lamas (ed.), El género: la construcción cultural de la diferencia sexual. México: PUEG, pp. 265-302.

Seri, R. y Farré, I. (2018). El espacio de las mujeres arquitectas graduadas en Rosario y sus producciones en las publicaciones disciplinares. En VIII Encuentro de docentes, investigadores en Historia del diseño, la arquitectura y la ciudad. Facultad de Arquitectura,

Urbanismo y Diseño, Universidad Nacional de Córdoba, Argentina.

Topolsky, J. (1992). Metodología de la historia. Madrid: Cátedra.

Trebisacce, C. (2016). Una historia crítica del concepto de experiencia de la epistemología feminista. Cinta moebio, 57, pp. 285-295. DOI: 10.4067/ S0717-554X2016000300004.

Daldi, N. S. (2018). Arquitectas. Estrategias y obstáculos de inserción de las primeras mujeres al campo de la Arquitectura argentina (primera mitad del siglo xx). Hábitat y Sociedad, 11, 15-29.

<http://dx.doi.org/10.12795/HabitatySociedad.2018.i11.02> 


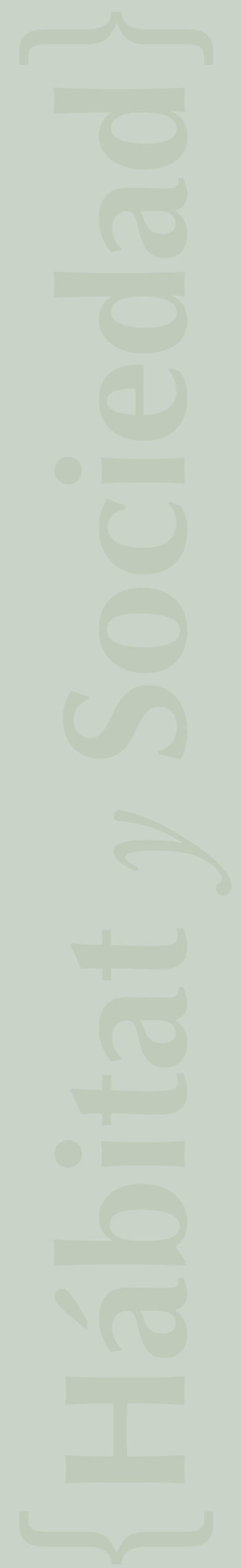

